\title{
(6) OPEN ACCESS \\ Antiepileptic drug treatment of rolandic epilepsy and Panayiotopoulos syndrome: clinical practice survey and clinical trial feasibility
}

\author{
Louise C Mellish, ${ }^{1}$ Colin Dunkley, ${ }^{2}$ Colin D Ferrie, ${ }^{3}$ Deb K Pal ${ }^{1}$
}

\section{- Additional material is published online only. To view please visit the journal online (http://dx.doi.org/10.1136/ archdischild-2013-304211). \\ ${ }^{1}$ King's College London, London, UK \\ ${ }^{2}$ Sherwood Forest Hospitals, Notts, UK \\ ${ }^{3}$ Department of Paediatric Neurology, Leeds General Infirmary, Leeds, UK \\ Correspondence to \\ Professor Deb K Pal, \\ Department of Basic and Clinical Neuroscience, King's College London, Institute of Psychiatry, Psychology \& Neuroscience, London SE5 8AF, UK; \\ deb.pal@kcl.ac.uk}

Received 26 February 2014 Revised 1 August 2014 Accepted 4 August 2014 Published Online First 8 September 2014
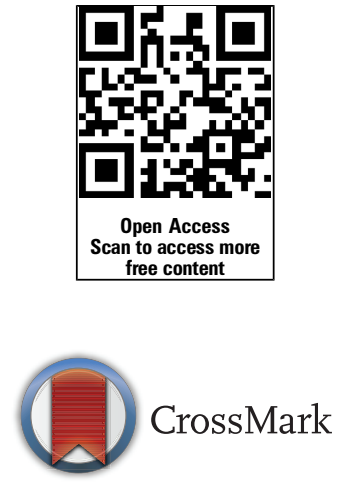

To cite: Mellish $\mathrm{LC}$, Dunkley C, Ferrie CD, et al. Arch Dis Child

2015; 100:62-67.

\section{ABSTRACT}

Background The evidence base for management of childhood epilepsy is poor, especially for the most common specific syndromes such as rolandic epilepsy (RE) and Panayiotopoulos syndrome (PS). Considerable international variation in management and controversy about non-treatment indicate the need for high quality randomised controlled trials (RCT). The aim of this study is, therefore, to describe current UK practice and explore the feasibility of different RCT designs for RE and PS.

Methods We conducted an online survey of 590 UK paediatricians who treat epilepsy. Thirty-two questions covered annual caseload, investigation and management practice, factors influencing treatment, antiepileptic drug preferences and hypothetical trial design preferences.

Results 132 responded (22\%): $81 \%$ were paediatricians and $95 \%$ at consultant seniority. We estimated, annually, 751 new RE cases and 233 PS cases. Electroencephalography (EEG) is requested at least half the time in approximately $70 \%$ of cases; MRI brain at least half the time in $40 \%-65 \%$ cases and neuropsychological evaluation in $7 \%-8 \%$. Clinicians reported non-treatment in $40 \%$ : main reasons were low frequency of seizures and parent/child preferences. Carbamazepine is the preferred older, and levetiracetam the preferred newer, RCT arm. Approximately one-half considered active and placebo designs acceptable, choosing seizures as primary and cognitive/behavioural measures as secondary outcomes.

Conclusions Management among respondents is broadly in line with national guidance, although with possible overuse of brain imaging and underuse of EEG and neuropsychological assessments. A large proportion of patients in the UK remains untreated, and clinicians seem amenable to a range of RCT designs, with carbamazepine and levetiracetam the preferred active drugs.

\section{INTRODUCTION}

Epilepsy affects 63400 young people under 18 years of age in the UK. ${ }^{1}$ Seizures represent one of the top five avoidable reasons for admission of children to emergency departments in the UK. ${ }^{2}$ Aside from seizures, cognitive and behavioural comorbidities cause a substantial impact affecting about two-thirds of children with epilepsy. ${ }^{3}$ Indeed, the comorbidity-associated burden may outweigh that of the seizures themselves ${ }^{4}$ and in some epilepsies, comorbidities are stronger predictors of quality-of-life than seizures. ${ }^{5}$ Hence, the comprehensive management of epilepsy necessitates the recognition and management of individual epilepsy syndromes and their specific comorbidities.

\section{What is already known on this topic?}

- UK management of rolandic epilepsy and Panayiotopoulos syndrome are not well known and there is limited scientific basis for drug treatment or non-treatment.

- Paediatric opinion towards clinical trial designs is also unknown and important to assess prior to further planning.

\section{What this study adds?}

- There are suggested patterns of underuse of EEG and neuropsychological assessment and overuse of brain MRI; $40 \%$ of patients are routinely untreated.

- Half the respondents would be open to head-to-head or active versus non-active design or placebo-controlled clinical trials with carbamazepine and levetiracetam as the preferred active treatments.

There are almost 40 electroclinical epilepsy syndromes defined by constellations of seizure type(s), age of onset, electroencephalography (EEG) and clinical features, each requiring individual assessment and management. The evidence base for management of childhood epilepsies in the UK is detailed in the National Institute of Health and Care Excellence (NICE) Guidelines ${ }^{6}$ and by its Scottish equivalent, the Scottish Intercollegiate Guidelines Network (SIGN). ${ }^{7}$ This guidance provides a care standard framework against which a recent national 'Epilepsy12' audit of childhood epilepsy care was conducted. ${ }^{8}$ However, the evidence base for antiepileptic drug treatment in these guidelines remains poor for many common childhood epilepsies, consisting of only a few high-quality randomised controlled trials (RCT). ${ }^{9}$ Much of the evidence available to NICE is extrapolated from heterogeneous studies of seizures mixing epilepsy types and age groups,${ }^{10}$ with uncertain applicability to well-defined childhood epilepsy syndromes. ${ }^{11}$ Furthermore, while there is now an emerging idea of how childhood epilepsy as a whole is managed in the $\mathrm{UK}^{8}{ }^{8}$ there is little detail about the variation in management of specific epilepsy syndromes and paediatricians' familiarity with them. Hence, a more detailed survey of practice is justified. 
In the absence of solid RCT evidence, there is widespread historical and geographical variation in recommendations for the drug management of specific epilepsies. In childhood, rolandic epilepsy (RE, sometimes known as Benign Epilepsy with CentroTemporal Spikes or BECTS), a focal epilepsy, is the most common syndrome, estimated to constitute $8 \%-25 \%$ of all childhood epilepsies ${ }^{12}$ and diagnosed in $9 \%$ of children with epilepsy in the recent Epilepsy 12 audit. ${ }^{8} \mathrm{RE}$, along with the closely related Panayiotopoulos syndrome (PS), ${ }^{13}$ are part of the group of non-lesional focal epilepsies (box 1). Textbooks and older 'expert opinion' often advised conservative management of RE, that is, with no antiepileptic drugs (AED) ${ }^{14-17}$ but neither the extent to which this conservative management is followed, nor its scientific rationale, ${ }^{14}{ }^{18} 19$ are known. Moreover, recent experimental evidence suggests that focal EEG spikes may disrupt simultaneous regional brain function ${ }^{20}$ and might also impair long-term learning ${ }^{21}$ and memory consolidation in sleep, ${ }^{22}$ prompting a re-evaluation of the 'benign' nature of this group of epilepsies.

When treated, carbamazepine and lamotrigine are recommended as first-line monotherapy by NICE (see table 1) and others, ${ }^{6} 1726$ although the evidence base is acknowledged to be poor, ${ }^{27-29}$ and there are theoretical risks of carbamazepine exacerbating the seizures or EEG abnormality ${ }^{30}{ }^{31}$ and also speech production. ${ }^{32}$ International practice beyond the UK

\section{Box 1 Clinical Description of RE and PS}

\section{Rolandic epilepsy}

Rolandic epilepsy (RE), also known as Benign Epilepsy of Childhood with Centro-Temporal Spikes (BECTS), is the most common epilepsy in childhood, with an incidence of up to 21 per 100000 children aged 15 years and under. ${ }^{12}$ The onset of seizures is between 3 and 12 years, and remission almost always occurs by adolescence. Seizures typically occur during sleep or drowsiness, are brief and involve unilateral sensorimotor symptoms (eg, numbness, tingling, drooling) of the pharynx, tongue, face, lips and sometimes hand. The affected side may alternate and seizures may infrequently become secondarily generalised. Neurodevelopmental disorders are very common $(40 \%)$ and include speech sound disorder, language impairment and reading disability, all of which usually precede seizures and aggregate among relatives. ${ }^{23}{ }^{24}$ Attention deficit hyperactivity disorder (ADHD) and migraine without aura are also strong but less frequent $(10 \%)$ associations. ${ }^{25}$

\section{Panayiotopoulos syndrome}

In Panayiotopoulos syndrome (PS), seizure semiology involves prominent autonomic symptoms. At onset, nausea, retching and vomiting are characteristic, often accompanied by other autonomic symptoms, such as pupillary changes, pallor/flushing, alterations in heart rate, breathing irregularities and temperature instability. ${ }^{13}$ Syncope-like episodes may occur. Impairment of consciousness develops as the seizure progresses, often accompanied by eye and head deviation. Seizures may end in hemi or generalised convulsions. Two-thirds of seizures occur during sleep. Seizures are often prolonged, most lasting over $10 \mathrm{~min}$ and many over $30 \mathrm{~min}$ (autonomic, non-convulsive status epilepticus). Potentially life-threatening cardiorespiratory arrest has been described in PS. As in RE, cognitive and behavioural difficulties have been associated with PS. Prognosis for remission of seizures is excellent.
Table 1 NICE recommendations for management of RE/PS (rolandic epilepsy/ Panayiotopoulos Syndrome)

NICE recommendations for rolandic epilepsy (BECTS)/Panayiotopoulos Syndrome

- Diagnosis and investigation by paediatrician with expertise

- Investigation to include EEG confirmation of electroclinical syndrome

- Neuroimaging not usually indicated in BECTS

- 'Children, young people and adults with epilepsy should be given information about their seizure type(s) and epilepsy syndrome, and the likely prognosis.'

- 'Discuss with the child or young person, and their family and/or carers, whether AED treatment for BECTS spikes, Panayiotopoulos syndrome... is indicated.'

- 'Be aware that carbamazepine and oxcarbazepine may exacerbate or unmask continuous spike and wave during slow sleep, which may occur in some children with RE/BECTS.'

\begin{tabular}{lll}
\hline First-line AEDs & Adjunctive AEDs & $\begin{array}{l}\text { Other AEDs considered } \\
\text { in tertiary care }\end{array}$ \\
\hline $\begin{array}{l}\text { Carbamazepine } \\
\text { Lamotrigine }\end{array}$ & Carbamazepine & Eslicarbazepine acetate \\
Levetiracetam & Clobazam & Lacosamide \\
Oxcarbazepine & Gabapentin & Phenobarbital \\
Sodium valproate & Lamotrigine & Phenytoin \\
& Levetiracetam & Pregabalin \\
& Oxcarbazepine & Tiagabine \\
& Sodium valproate & Vigabatrin \\
& Topiramate & Zonisamide
\end{tabular}

AEDs, antiepileptic drugs; BECTS, Benign Epilepsy with CentroTemporal Spikes; NICE, National Institute of Health and Care Excellence.

diverges widely, ${ }^{29} 33$ as does expert opinion on the subject. ${ }^{34}$ Sulthiame is considered first-line in Germany, Austria and Israel, ${ }^{35}{ }^{36}$ although it may have adverse effects on cognition; ${ }^{37}$ sodium valproate in France; while levetiracetam is popular in the USA. Thus, the rationale for treatment versus non-treatment is not established and the evidence base in favour of specific AEDs is unknown amid widespread national and international variation in practice. This equipoise scenario sets the stage for an RCT, for which it would be important to learn the treatment preferences of physicians likely to recruit to such a trial, in order to assess various aspects of design feasibility. The purpose of this paper is, therefore, (1) to examine current clinical practice for RE and PS in relation to NICE guidance, specifically asking what proportion of patients are routinely not treated and (2) to explore the feasibility of alternative syndrome-specific RCT designs for RE and PS. We specifically address the questions of clinicians' attitudes towards placebo-controlled designs, and which AEDs would be either preferred or unpopular comparators.

\section{METHODS}

\section{Survey}

We designed a questionnaire targeting UK paediatricians with clinical responsibility for epilepsy (see online supplementary appendix 1). Thirty-two questions covered areas specifically related to RE and PS: (1) annual caseload of new patients; (2) investigation and management practice; (3) factors influencing AED treatment versus no treatment; (4) AED preferences between 'older' (before 1980) and 'newer' drugs; (5) theoretical RCT design and outcome preferences. Preference items were scored on a 5 -point Likert scale. We piloted a paper version among five paediatric epilepsy specialists and then created a modified online, forced-choice version using SurveyMonkey.

\section{Participants}

We distributed the online survey to the 282 Epilepsy-12 audit leads $^{8}$ and to 308 members of the British Paediatric Neurology 
Association between November 2012 and December 2012. Respondents could only reply once and this was checked by names and internet protocol addresses. We sent weekly email reminders before closing the weblink after 4 weeks. This professional survey was deemed exempt from ethics approval.

\section{Analysis}

Results were available both as summary data and as raw response files. We edited out inconsistent responses using the raw data. We summarised and visualised results using a spreadsheet tool.

\section{RESULTS}

\section{Characteristics of respondents}

There was a total of 132 respondents from the 590 individuals contacted with valid email addresses, a response rate of $39 \%$ from Epilepsy-12 audit leads and 22\% overall. With weekly email prompts, 74 responded in the first week, another 34 in the second week and the remainder responded over the following fortnight. Fifty-three percent of respondents were general paediatricians with special expertise in epilepsy; 17\% were general, community or neurodisability paediatricians; $19 \%$ were paediatric neurologists. In terms of seniority, 95\% were consultants; $5 \%$ associate specialists. Fifty-three percent of respondents were men. Age-wise, 11\% of respondents were under 40 years; $54 \%$ were $41-50$ years; $35 \%$ over 50 years.

\section{Caseload}

The majority (90\%) of clinicians reported diagnosing six or fewer new RE and six or fewer PS cases annually; by summating monthly individual case loads, we estimated an annual total of 751 new RE cases and 233 new PS cases from the 132 UK respondents.

\section{Investigation}

EEG is the most often requested investigation (figure 1). Most clinicians only infrequently request CT or MRI brain scans. Only $7 \%-8 \%$ of clinicians request neuropsychological assessments in RE or PS.

\section{Treatment}

Forty percent of RE and PS cases are never treated with regular AEDs. Both seizure frequency/severity and parental/child preference were judged important factors influencing the decision not to prescribe AED (figure 2).

\section{AED preference in RCTs}

AED preferences were almost identical for RE and PS (figure 3). Carbamazepine was overwhelmingly rated as the most preferred active comparator for an RCT and also the preferred 'older' AED; sodium valproate was the second most preferred AED; no other AEDs were indicated as first choice by $>10 \%$ of respondents. Among the newer AEDs, respondents indicated a slight preference for levetiracetam over lamotrigine as active comparator, with no other popular first-line choices $>10 \%$. About $10 \%$ of respondents would object to ethosuximide, benzodiazepines, phenobarbital or phenytoin as an active treatment arm.

\section{Attitudes to clinical trial design}

In RE, just over one-half of respondents (55\%) would recruit to an RCT comparing either two active treatments, $48 \%-49 \%$ to active versus no active treatment design and $41 \%$ to active versus placebo, regardless of the preceding number of seizures ( $<3$ or $\geq 3$ in 6 months). In PS, there was a greater preference

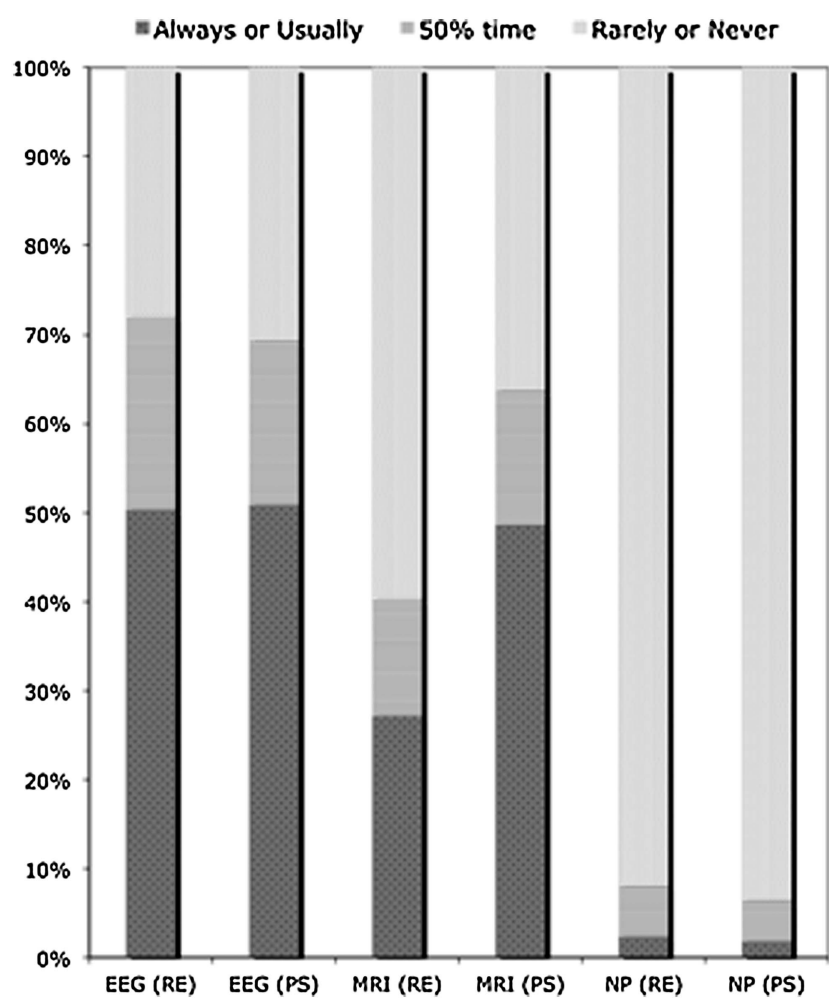

Figure 1 Use of investigations in rolandic epilepsy (RE) and Panayiotopoulos Syndrome (PS) expressed as percentage of respondents: electroencephalography (EEG); brain MRI; neuropsychological assessment (NP).

for a two active drug design (59\%) than a trial with no active treatment $(44 \%)$ or placebo $(38 \%)$. The great majority $(82 \%$ for RE and $79 \%$ for PS) chose seizure remission as the preferred primary outcome in a RE trial, with close to two-thirds (73\% for RE and $60 \%$ for PS) choosing cognitive or broad quality of life measures as a secondary outcome.

\section{DISCUSSION}

This is the first physician survey regarding the common epilepsy syndromes of childhood RE and PS. The results indicate first, that the pattern of investigations requested for patients with these syndromes appears broadly appropriate and in line with NICE guidance, although with a few unexplained observations.

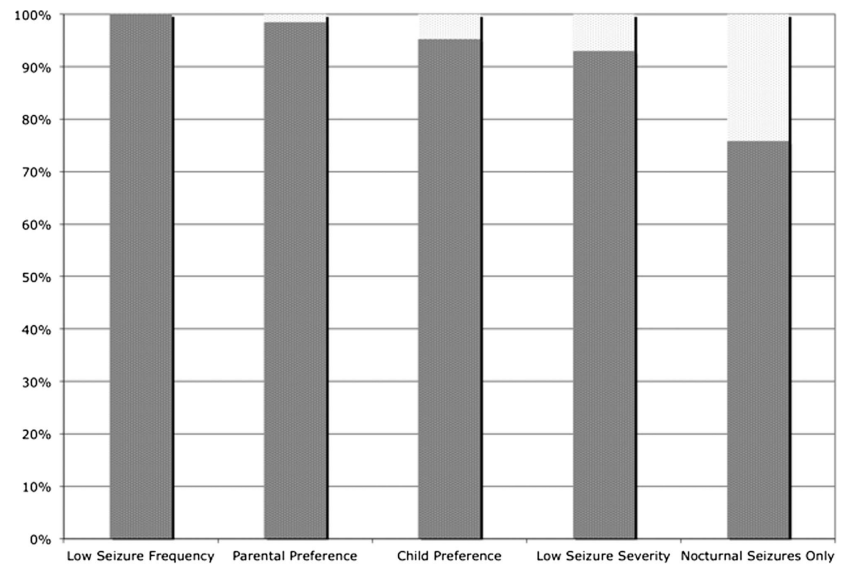

Figure 2 Factors rated as quite or very important influencing a no-treatment decision in rolandic epilepsy, expressed as percentage of respondents (data for Panayiotopoulos Syndrome very similar). 

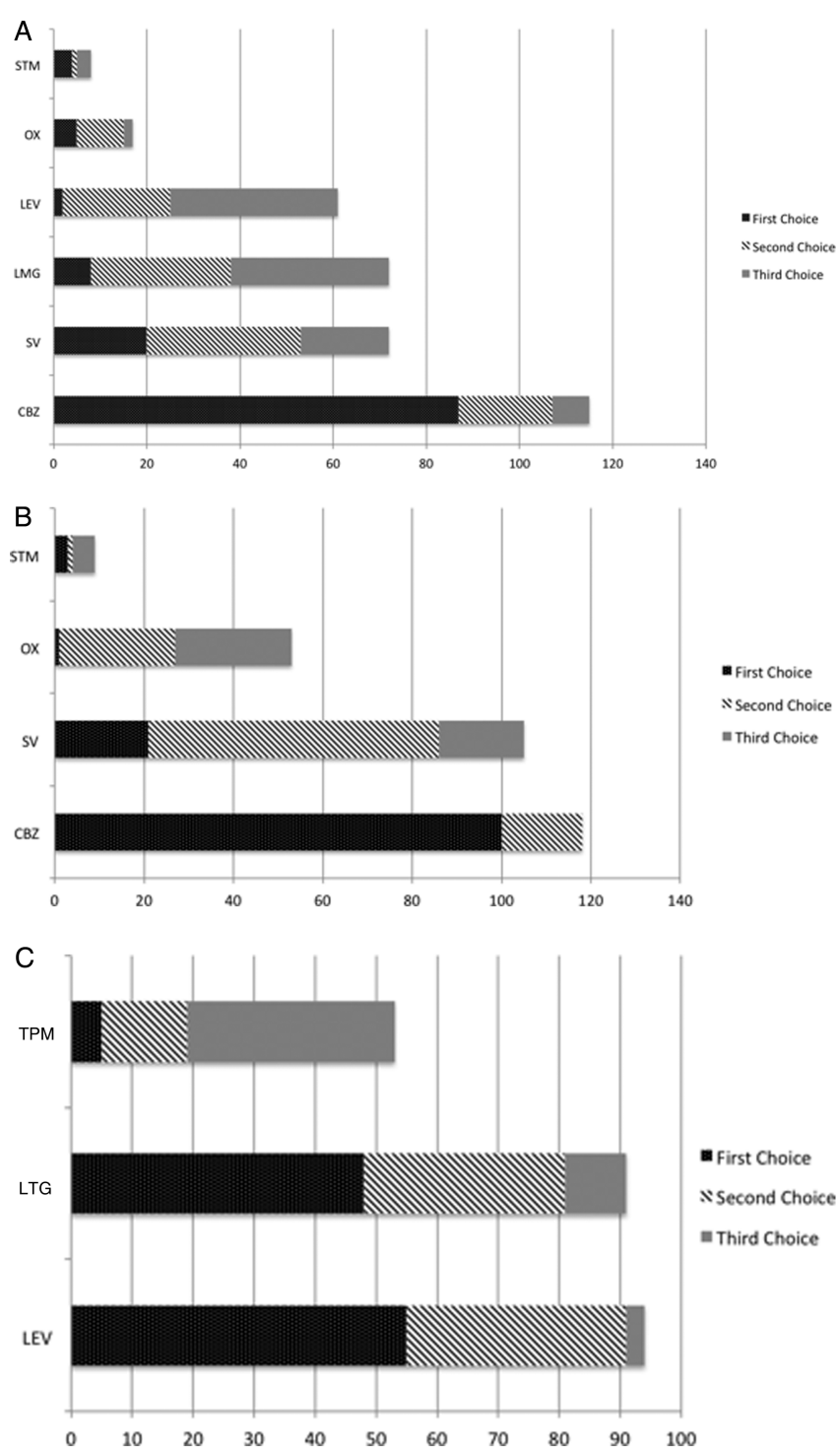

Figure 3 Clinicians' preferred choices of (A) all; (B) older and (C) newer treatments. STM, Sulthiame; OX, oxcarbazepine; LEV, levetiracetam; LTG, lamotrigine; SV, sodium valproate; CBZ, carbamazepine; TPM, topiramate.

Second, a large proportion of children with RE and PS remains untreated, reportedly influenced by a number of medical and social factors. Third, clinicians seem amenable to both placebocontrolled and head-to-head RCTs to address the question of drug superiority; they chose conventional primary seizure outcomes and secondary cognitive/behavioural outcomes. Carbamazepine would be the preferred older AED in a hypothetical RCT and levetiracetam, narrowly, the preferred newer AED. Inclusion of older AEDs, such as ethosuximide, phenobarbital, phenytoin and benzodiazepines, would deter recruitment among a significant minority. Last, response data support the existence of a sufficiently large sampling frame of new-onset RE and PS cases to construct an RCT in the UK.

The overall pattern of reported investigations appears broadly appropriate aside from some unexplained observations. For RE and PS, NICE and SIGN recommend EEG at diagnosis, with follow-up ambulatory, sleep or video EEG if needed; MRI brain scans are not recommended as first-line investigations because of the non-lesional nature of these epilepsies. In our survey, $70 \%$ of clinicians reported using EEG as a first-line investigation at least half the time (figure 1); those who do not may possibly not have ready access to it-Epilepsy 12 suggests that over $40 \%$ do not have local access to these facilities. ${ }^{8}$ Whatever the explanation, it is debatable whether a diagnosis of epilepsy, especially a 'benign' syndrome, should be made without benefit of EEG. Brain MRI was requested in approximately $25 \%$ of RE cases and $50 \%$ of PS cases. While the clinical history usually obviates the need for brain imaging in RE (especially in the presence of a suggestive EEG), and brain imaging in RE can generate incidental findings, ${ }^{38}$ variable clinical features can make an early diagnosis more challenging in PS and might explain the higher frequency of imaging requests. ${ }^{39}$ Nevertheless, the overall use of MRI appears unusually high for these non-lesional syndromes and is worthy of further investigation. The very low use of neuropsychological assessments $(<10 \%)$ is striking given the well-documented occurrence of language, literacy and attentional comorbidities in $\mathrm{RE}^{2324}$ and their impact on educational achievement and quality of life. ${ }^{5}$ Although this topic is not covered by professional guidelines, it merits investigation as it could reflect correctable factors, for example, either poor local access to assessment services, low awareness about the high prevalence of treatable comorbidities or referral to alternative services, for example, school educational psychologist.

A large proportion of patients with RE and PS are not routinely treated with AEDs. Survey respondents indicated that low seizure frequency, parental and child preference, low seizure severity and nocturnal seizure predominance were the most important factors influencing a policy of no treatment (figure 2). These same factors have been cited as reasons not to treat. ${ }^{14-17} 19$ However, we neither know whether untreated patients are missing important benefits of treatment, nor whether treated patients are needlessly suffering adverse effects. For example, we do not know whether regular AED treatment overall mitigates or exacerbates the frequent cognitive and attentional comorbidities. Suggestions that interictal focal EEG discharges led to transitory regional cognitive dysfunction, ${ }^{20}$ have now been augmented by experimental evidence in the rat model that interictal EEG discharges can impair long-term learning. ${ }^{21}$ Meanwhile, emerging data suggest that interictal spikes even in benign focal epilepsies can impair memory consolidation in sleep. $^{22}$ On the other hand, there is also evidence that carbamazepine can worsen speech production ${ }^{32}$ and sulthiame may worsen cognitive function in RE. ${ }^{37}$ Quality of life appears to suffer regardless of seizure number, suggesting that clinical severity has a loose correlation with child and family impact. ${ }^{18}$ The no-treatment practice does not take into account the costs associated with emergency room attendance and hospital admission for unprevented seizures. These various uncertainties point to the necessity for any RCT to address the rationale for nontreatment. Such a trial could also be designed to evaluate the superiority of active comparators.

The evidence base for treatment choice in both RE and PS is acknowledged to be poor, ${ }^{9}$ with only three relevant Class III studies (eg, open-label) suggesting that carbamazepine and sodium valproate are 'possibly' effective, and levetiracetam, oxcarbamazepine, gabapentin and sulthiame 'potentially' effective as initial monotherapy. ${ }^{27-29} 40$ There are many other class IV studies (ie, observational) that cannot be used to support guidelines. ${ }^{28} 3741-43$ Among active comparators in the survey, carbamazepine is clearly the referent first-line AED for RE/PS trials (figure $3 \mathrm{~A}$ and $\mathrm{B}$ ). Despite concerns about carbamazepine worsening electroclinical features and triggering continuous spikes in slow-wave sleep, ${ }^{30}$ only $6 \%-8 \%$ respondents indicated its inclusion would deter recruitment of patients to an RCT. Critics feel 
that carbamazepine has not been properly evaluated in childhood epilepsy: the Standard and New Antiepileptic Drugs (SANAD) trial (upon which NICE guidelines are based) included few patients with RE/PS $(n=24)$ and mixed focal seizures of diverse epilepsy syndromes, for example, temporal lobe epilepsy together, possibly masking pertinent data. ${ }^{10} 11$ Although lamotrigine (along with carbamazepine) is considered by NICE as a firstline and sodium valproate as a second-line agent, we observe the reverse preferences in our survey (figure $3 \mathrm{~A}$ ). Looking only at newer AEDs, levetiracetam emerges as the first choice, ahead of lamotrigine (figure 3C). Neither topiramate, oxcarbazepine nor sulthiame (not licensed in the UK) was commonly chosen as a possible comparator; and much older AEDs, for example, phenobarbital, phenytoin, ethosuximide and benzodiazepines were not acceptable to about $10 \%$ and there is little scientific rationale to include them in a future trial in developed countries. Since older AEDs (carbamazepine and sodium valproate here) are known to have comparable efficacy in childhood, ${ }^{44}$ there would only be merit in comparing referent AED with newer $\mathrm{AED}(\mathrm{s})$. Levetiracetam is the obvious newer comparator AED because: (1) it has not been adequately evaluated as monotherapy in the paediatric population despite widespread off-license use $;^{35} 45$ (2) it is the most favoured newer AED in this survey; (3) it has come off-patent since the time of the European consensus statement and will therefore be an economically viable comparator to older AEDs; ${ }^{46}$ (4) lamotrigine monotherapy has been compared with carbamazepine ${ }^{47}$ and sodium valproate. ${ }^{48}$ However, outside the UK, clinicians may prefer alternative comparators: European 'expert opinion' considers sodium valproate drug of choice for RE and carbamazepine only as 'sometimes appropriate'. ${ }^{34}$ German-speaking countries, Japan and Israel favour sulthiame, ${ }^{33}$ although concerns remain over its cognitive adverse effects. ${ }^{37}$

While non-treatment is common practice, and we conclude the need to conduct an RCT to determine whether this is justified, would clinicians countenance a trial design with no active arm? The survey results are encouraging: approximately one-half of respondents would recruit either to a head-to-head, active versus non-active or to a placebo-controlled RCT in RE. Respondents were equally prepared to randomise after either less than, or more than, two seizures in 6 months. However, there was a more pronounced preference towards active comparators in PS. Thus, non-active or placebo-controlled designs can be considered feasible options in trial design.

A cross-sectional survey has several limitations and is only a proxy for actual observation of practice. The response rate was relatively low and the results may reflect selection bias towards respondents who conform more closely to norms and guidelines, giving a more positive impression of management. Clinicians who see many patients may also be more inclined to respond, inflating the estimates of caseload. Clinicians motivated to complete surveys may also, as a group, be more amenable towards RCTs, thus, overestimating true potential recruitment. This survey principally targeted general paediatricians who have primary responsibility for childhood epilepsy in their district or audit zone, all of whom were involved in the recent Epilepsy12 audit. Since this is the group that has primary contact with children with RE or PS, they would be the clinicians who are likely to recruit such children into an RCT and, as such, their opinions are important to determine feasibility. The response rate among the general paediatrician group was 39\% and even if we only double the number of reported cases to estimate the total number seen within audit zones, there might be $1500 \mathrm{RE}$ and 500 PS ascertainable annual cases. This is a higher number compared with the Epilepsy12 audit, which estimated 340 annual RE cases. ${ }^{8}$ The discrepancy between the figures from the two studies might be due to the low rate of syndromic diagnoses that were made by clinicians participating in Epilepsy12 or due to over-reporting of local caseloads in our survey, for example, if there was shared care of the same patient between a paediatrician and a paediatric neurologist. Approximately half the respondents indicated a willingness to recruit to an RCT, still yielding a sufficient sampling frame to consider further planning.

Overall, the question whether or not to treat children with non-lesional focal epilepsy syndromes like RE and PS remains unanswered. Furthermore, supporting evidence to validate the use of specific AEDs for either RE or PS is limited despite widespread variation in practice. Encouragingly, this survey demonstrates: (1) there is a sufficient UK network of clinicians who see a large number of these patients; (2) these clinicians are willing to resolve these issues of treatment and non-treatment uncertainty through an RCT; (3) a non-active or placebo arm would be acceptable, and carbamazepine and levetiracetam would be the preferred (and most informative) active comparators. We suggest further discussion and planning of trial design features such as the issue of combining RE and PS, placebo-control, active arms, blinding, outcome measures, logistics and so on, within a larger national or international multidisciplinary study group.

Contributors DKP conceived the study. LCM designed the questionnaire with input from all authors. LCM and CD administered the survey. LCM and DKP analysed the results in discussion with CD and CDF. LCM wrote the first draft and subsequent drafts were edited by all authors.

Funding Writing of this manuscript was supported by the European Union Marie Curie International Reintegration Award of the Seventh Framework Programme (DKP); Waterloo Foundation (DKP); Charles Sykes Epilepsy Research Trust (DKP); NIHR Specialist Biomedical Research Centre for Mental Health of South London and Maudsley NHS Foundation Trust (DKP).

\section{Competing interests None.}

Provenance and peer review Commissioned; externally peer reviewed.

Data sharing statement The full version of the questionnaire is available from corresponding author.

Open Access This is an Open Access article distributed in accordance with the Creative Commons Attribution Non Commercial (CC BY-NC 3.0) license, which permits others to distribute, remix, adapt, build upon this work non-commercially, and license their derivative works on different terms, provided the original work is properly cited and the use is non-commercial. See: http://creativecommons.org/ licenses/by-nc/3.0/

\section{REFERENCES}

1 Joint Epilepsy Council. Epilepsy prevalence, incidence and other statistics. www.jointepilepsycouncil.org.uk; Leeds 2011.

2 Saxena S, Bottle A, Gilbert R, et al. Increasing short-stay unplanned hospital admissions among children in England; time trends analysis '97-'06. PLOS ONE 2009;4:e7484.

3 Camfield C, Breau L, Camfield P. Assessing the impact of pediatric epilepsy and concomitant behavioral, cognitive, and physical/neurologic disability: impact of childhood neurologic disability scale. Dev Med Child Neurol 2003;45:152-9.

4 Institute of Medicine (US). Committee on the public health dimensions of the epilepsies board on health sciences policy. In: England MJ, Liverman CT, Schultz AM, Strawbridge LM. eds. Epilepsy across the spectrum. Promoting health and understanding. Washington, DC: Institute of Medicine of the National Academies, 2012:572.

5 Connolly AM, Northcott E, Cairns DR, et al. Quality of life of children with benign rolandic epilepsy. Pediatr Neurol 2006;35:240-5.

6 The National Institute for Health and Clinical Excellence. The epilepsies: the diagnosis and management of the epilepsies in adults and children in primary and secondary care. 2012. Retrieved from http://www.nice.org.uk/cg137, 2014.

7 Scottish Intercollegiate Guidelines Network. The diagnosis and management of the epilepsies in adults and children in primary and secondary care: a national clinical guideline. 2005. Retrieved from www.sign.ac.uk/guideline70, 2014. 
8 Dunkley C. Epilepsy12 national report; United Kingdom collaborative clinical audit of health care for children and young people with suspected epileptic seizures. Royal College of Paediatrics and Child Health, 2012.

9 Glauser T, Ben-Menachem E, Bourgeois B, et al. Updated ILAE evidence review of antiepileptic drug efficacy and effectiveness as initial monotherapy for epileptic seizures and syndromes. Epilepsia 2013;54:551-63.

10 Marson AG, Al-Kharusi AM, Alwaidh M, et al. The SANAD study of effectiveness of carbamazepine, gabapentin, lamotrigine, oxcarbazepine, or topiramate for treatment of partial epilepsy: an unblinded randomised controlled trial. Lancet 2007;369:1000-15.

11 Ferrie CD, Grunewald RA, Livingston JH, et al. Treatment of newly diagnosed epilepsies: the shortcomings of SANAD. Dev Med Child Neurol 2008;50:247

12 Cavazzuti GB. Epidemiology of different types of epilepsy in school age children of Modena, Italy. Epilepsia 1980;21:57-62.

13 Panayiotopoulos CP, Michael M, Sanders S, et al. Benign childhood focal epilepsies: assessment of established and newly recognized syndromes. Brain 2008; 131:2264-86.

14 Ambrosetto G, Tassinari CA. Antiepileptic drug treatment of benign childhood epilepsy with rolandic spikes: is it necessary? Epilepsia 1990;31:802-5.

15 Peters JM, Camfield CS, Camfield PR. Population study of benign rolandic epilepsy: is treatment needed? Neurology 2001;57:537-9.

16 Loiseau P, Duche B, Cohadon S. The prognosis of benign localized epilepsy in early childhood. Epilepsy Res Supp/ 1992;6:75-81.

17 Lerman P, Kivity S. Benign focal epilepsy of childhood. A follow-up study of 100 recovered patients. Arch Neurol 1975;32:261-4.

18 Modi AC, King AS, Monahan SR, et al. Even a single seizure negatively impacts pediatric health-related quality of life. Epilepsia 2009;50:2110-6.

19 Verrotti A, Latini G, Trotta $D$, et al. Population study of benign rolandic epilepsy: is treatment needed? Neurology 2002;59:476.

20 Aarts JH, Binnie CD, Smit AM, et al. Selective cognitive impairment during focal and generalized epileptiform EEG activity. Brain 1984;107(Pt 1):293-308.

21 Khan Ol, Zhao Q, Miller F, et al. Interictal spikes in developing rats cause long-standing cognitive deficits. Neurobiol Dis 2010;39:362-71.

22 Urbain C, Di Vincenzo T, Peigneux P, et al. Is sleep-related consolidation impaired in focal idiopathic epilepsies of childhood? A pilot study. Epilepsy Behav 2011;22:380-4.

23 Clarke T, Strug LJ, Murphy PL, et al. High risk of reading disability and speech sound disorder in rolandic epilepsy families: case-control study. Epilepsia 2007:48:2258-65.

24 Smith A, Kavros PM, Clarke T, et al. A neurocognitive endophenotype associated with Rolandic Epilepsy. Epilepsia 2012;53:705-11.

25 Clarke T, Baskurt Z, Strug LJ, et al. Evidence of shared genetic risk factors for migraine and rolandic epilepsy. Epilepsia 2009;50:2428-33.

26 Wirrell EC. Benign epilepsy of childhood with centrotemporal spikes. Epilepsia 1998;39(Suppl 4):S32-41.

27 Bourgeois B, Brown L, Pellock J, et al. Gabapentin (Neurontin) monotherapy in children with benign childhood epilepsy with centrotemporal spikes (BECTS): a 36 week double-blind placebo-controlled study. Epilepsia 1998;39:163.

28 Coppola G, Franzoni E, Verrotti A, et al. Levetiracetam or oxcarbazepine as monotherapy in newly diagnosed benign epilepsy of childhood with centrotemporal spikes (BECTS): an open-label, parallel group trial. Brain Dev 2007;29:281-4.
29 Rating D, Wolf C, Bast T. Sulthiame as monotherapy in children with benign childhood epilepsy with centrotemporal spikes: a 6 month randomized, double-blind, placebo-controlled study. Sulthiame Study Group. Epilepsia 2000:41:1284-8

30 Lerman P. Seizures induced or aggravated by anticonvulsants. Epilepsia 1986;27:706-10.

31 Deonna TW, Roulet E, Fontan D, et al. Speech and oromotor deficits of epileptic origin in benign partial epilepsy of childhood with rolandic spikes (BPERS). Relationship to the acquired aphasia-epilepsy syndrome. Neuropediatrics 1993;24:83-7.

32 Park JI, Kim SJ, Kim HG. Acoustic effects of carbamazepine in benign rolandic epilepsy. Epilepsy Behav 2005;7:468-71.

33 Gross-Selbeck G. Treatment of "benign" partial epilepsies of childhood, including atypical forms. Neuropediatrics 1995;26:45-50.

34 Wheless JW, Clarke DF, Arzimanoglou A, et al. Treatment of pediatric epilepsy: European expert opinion, 2007. Epileptic Disord 2007;9:353-412.

35 Borggraefe I, Bonfert M, Bast T, et al. Levetiracetam vs. sulthiame in benign epilepsy with centrotemporal spikes in childhood: a double-blinded, randomized, controlled trial (German HEAD Study). Eur J Paediatr Neurol 2013;17:507-14.

36 Rating D. Treatment in typical and atypical rolandic epilepsy. Epileptic Disord 2000;2:S69-72.

37 Wirrell E, Sherman EM, Vanmastrigt $R$, et al. Deterioration in cognitive function in children with benign epilepsy of childhood with central temporal spikes treated with sulthiame. J Child Neurol 2008;23:14-21.

38 Boxerman JL, Hawash K, Bali B, et al. Is Rolandic epilepsy associated with abnormal findings on cranial MRI? Epilepsy Res 2007;75:180-5.

39 Ferrie C, Caraballo R, Covanis A, et al. Panayiotopoulos syndrome: a consensus view. Dev Med Child Neurol 2006;48:236-40.

40 Andrade R, Garcia-Espinosa A, Machado-Rojas A, et al. [A prospective, open, controlled and randomised study of clobazam versus carbamazepine in patients with frequent episodes of Rolandic epilepsy]. Rev Neurol 2009;49:581-6.

41 Kossoff EH, Los JG, Boatman DF. A pilot study transitioning children onto levetiracetam monotherapy to improve language dysfunction associated with benign rolandic epilepsy. Epilepsy Behav 2007;11:514-17.

42 Kang $\mathrm{HC}$, Eun BL, Wu Lee $\mathrm{C}$, et al. The effects on cognitive function and behaviora problems of topiramate compared to carbamazepine as monotherapy for children with benign rolandic epilepsy. Epilepsia 2007;48:1716-23.

43 Tzitiridou M, Panou T, Ramantani G, et al. Oxcarbazepine monotherapy in benign childhood epilepsy with centrotemporal spikes: a clinical and cognitive evaluation. Epilepsy Behav 2005;7:458-67.

44 de Silva M, Macardle B, McGowan M, et al. Randomised comparative monotherapy trial of phenobarbitone, phenytoin, carbamazepine, or sodium valproate for newly diagnosed childhood epilepsy. Lancet 1996;347:709-13.

45 Arya R, Glauser TA. Pharmacotherapy of focal epilepsy in children: a systematic review of approved agents. CNS Drugs 2013;27:273-86.

46 Lane J. UCB faces patent cliff in 2012. 2012. http://www.pharmafield.co.uk/be/post/ 2012/03/05/UCB-faces-patent-cliff-in-2012.aspx

47 Gamble CL, Williamson PR, Marson AG. Lamotrigine versus carbamazepine monotherapy for epilepsy. Cochrane Database Syst Rev 2006;(1):CD001031.

48 Glauser TA, Cnaan A, Shinnar S, et al. Ethosuximide, valproic acid, and lamotrigine in childhood absence epilepsy. N Engl J Med 2010;362:790-9. 